Sir,

\section{Orbital venous-lymphatic malformation}

An orbital venous-lymphatic malformation is a rare congenital lesion, the clinical and radiological features of which have been highlighted by Liyanage et al. ${ }^{1}$ We present our surgical experience and histopathological description of a patient having a similar condition.

\section{Case report}

A 39-year-old roofer was seen with a 2-week history of an enlarging vascular conjunctival lesion in the medial
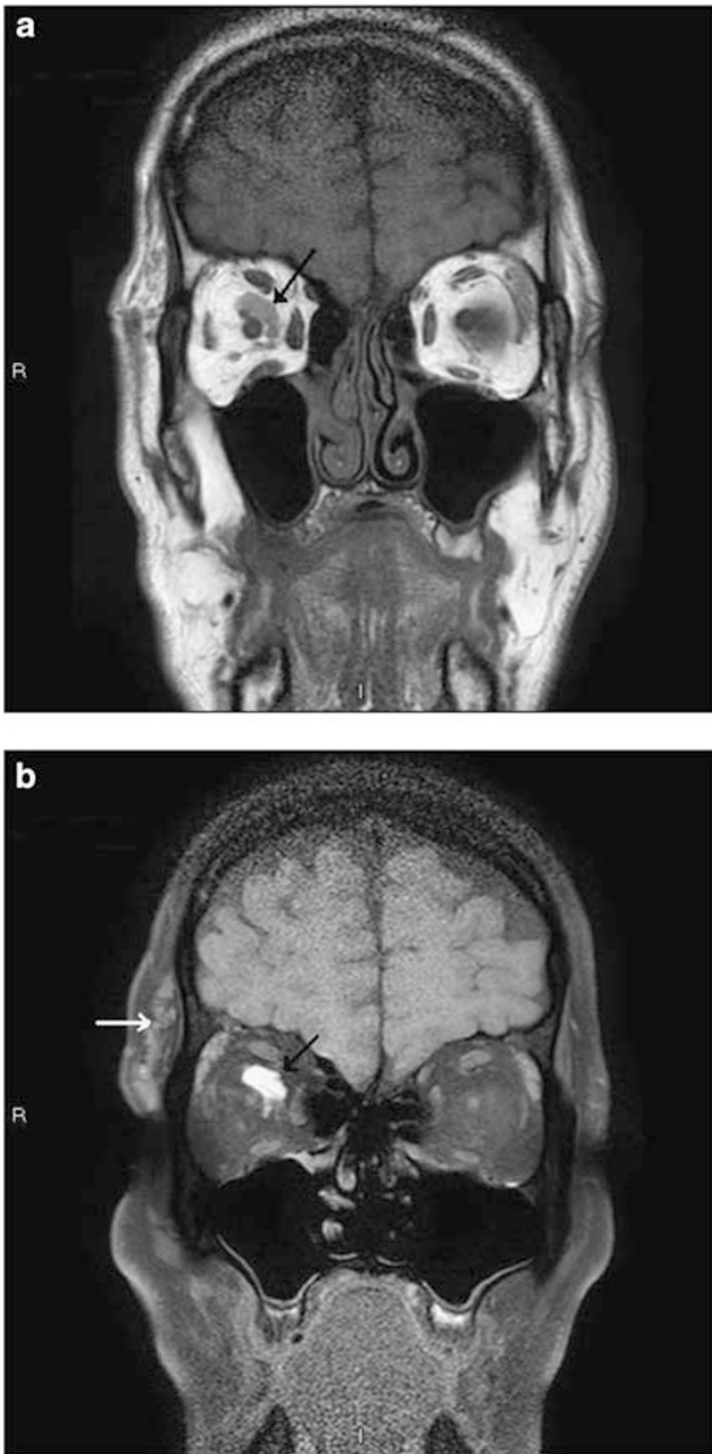

Figure 1 (a) T1-weighted coronal view showing an intracoronal large serpiginous soft tissue mass involving the superior aspect of the right orbit (black arrow). (b) T1-weighted coronal view after intravenous contrast showing an uptake of contrast in the right orbital soft tissue mass suggesting vascular/haemangiomatous lesion (black arrow). There is some vascular enhancement with some associated soft tissue in the subcutaneous aspect of the right temple (white arrow). This appears to communicate through a leash of vessels with the right orbital mass. canthal area and a diffuse swelling in the temporal area He also reported the presence of a superonasal anterior orbital swelling since birth. His vision, ocular movements, and position of the eyeball were unaffected. A magnetic resonance imaging (MRI) scan showed a serpiginous superior orbital mass having a vascular communication to the subcutaneous soft tissue mass on
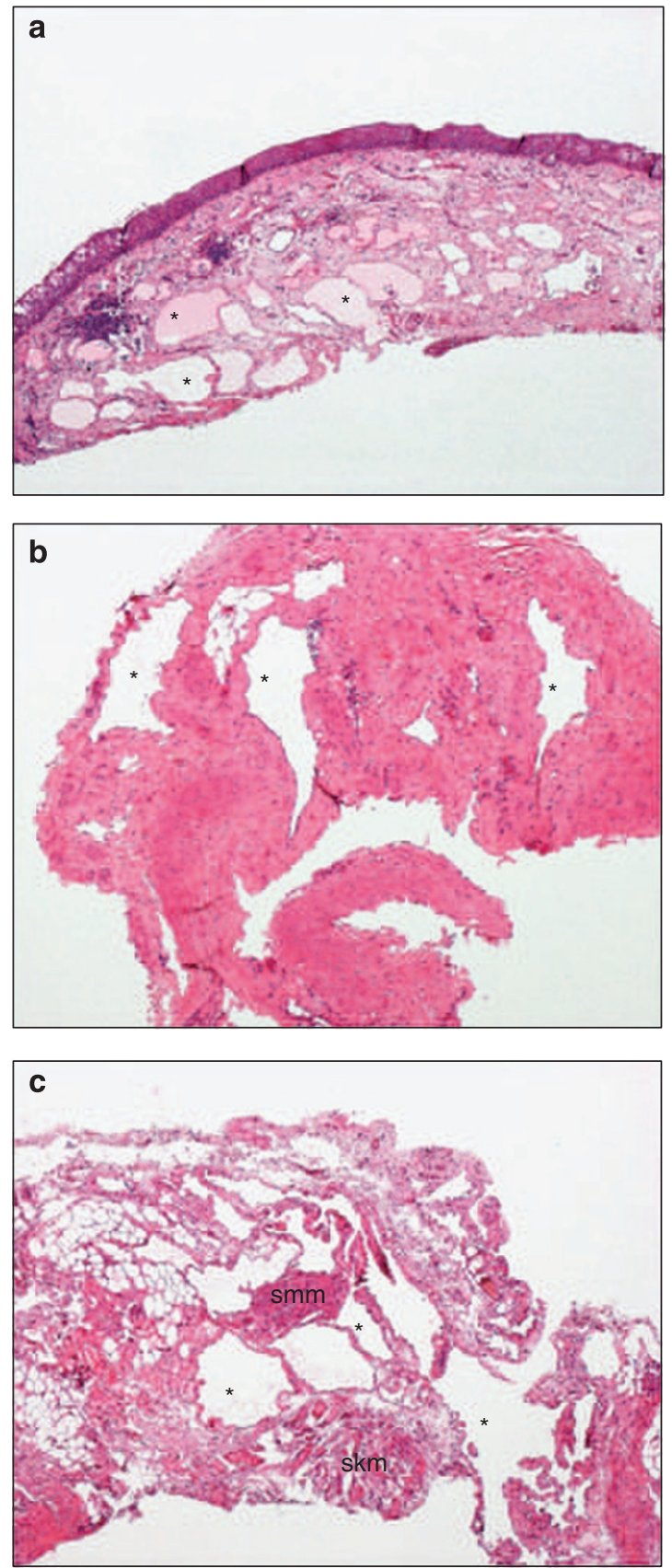

Figure 2 (a) Specimen from superficial plica showing conjunctival mucosa with numerous dilated lymphatic channels in the submucosa $\left({ }^{*}\right)$. H\&E $\times 40$. (b) Dense fibrous tissue from the upper fornix showing large dilated presumed lymphatic channels ${ }^{*}$ ). H\&E $\times 100$. (c) Further tissue from the upper fornix showing fat, skeletal muscle (skm), and smooth muscle (smm) permeated by lymphatic channels $\left({ }^{*}\right)$. H\&E, $\times 40$. 
the temple (Figure 1). The nature of the lesion was consistent with a vascular malformation or extensive orbital varix. A surgical biopsy was carried out to obtain a tissue diagnosis and partially debulk the visible part of the lesion. The lesion had strong adhesions making dissection difficult. A slow persistent ooze was noticed from the lesion throughout the surgery.

Histopathology showed numerous dilated interconnecting vascular channels in all the excised tissues, which included the mucosa and submucosa of the conjunctiva, Muller's muscle fibres, fat, and connective tissue (Figure 2). The ramifying abnormally dilated channels were in keeping with a lymphaticvenous malformation or lymphangioma. This corroborated with the clinical and radiological picture.

\section{Comment}

Surgical excision of lymphatic-venous malformations is often difficult because of the intricate architecture of these lesions and the risk of associated haemorrhage. ${ }^{2}$ Multiple surgical excisions may be required for a satisfactory result. Although complete surgical excision has been reported, cicatrisation and recurrence are known problems. ${ }^{3}$ Any surgery should be conservative and aimed at obtaining a tissue diagnosis and partial debulking. In our patient, the vision and ocular movements were well-preserved and any extensive surgery would have run a high risk of visual loss and diplopia.

There have been anecdotal reports of success with sclerosing agents such as OK-432 and sodium tetradecyl sulphate. ${ }^{4,5}$ A prospective study on six patients found sodium morrhuate $5 \%$ to be a very effective sclerosing agent. ${ }^{2}$ These approaches may be considered in suitable cases to limit surgical intervention.

\section{References}

1 Liyanage SE, Watson GMT, Wearne MJ. Late ocular manifestation of a childhood venous-lymphatic malformation. Eye 2008; 22: 1194-1195.

2 Schwarcz RA, Simon GJB, Cook T, Goldberg RA. Sclerosing therapy as first line treatment for low flow vascular lesions of the orbit. Am J Ophthalmol 2006; 141: 333-339.

3 Harris GJ, Sakol PJ, Bonavolonta G, De Conciliis C. An analysis of thirty cases of orbital lymphangioma: pathophysiologic considerations and management recommendations. Ophthalmology 1990; 97: 1583-1592.

4 Suzuki Y, Obana A, Gohto Y, Miki T, Otuka H, Inoue Y. Management of orbital lymphangioma using intralesional injection of OK-432. Br J Ophthalmol 2000; 84: 614-617.

5 Wojno TH. Sotradecol (sodium tetradecyl sulfate) injection of orbital lymphangioma. Ophthal Plast Reconstr Surg 1999; 15: 432-437.

V Chadha, MA Awan, P Gonzalez, P Cauchi, F Roberts and E Kemp

Department of Ophthalmology, Tennent Institute of Ophthalmology, Gartnavel General Hospital, Glasgow, UK

E-mail:vchdh@aol.com

Eye (2009) 23, 2265-2266; doi:10.1038/eye.2009.11; published online 13 February 2009
Sir,

RetCam compression artifact can mask plus disease

The current treatment of serious retinopathy of prematurity (ROP) requires accurate diagnosis of plus disease to optimize outcome. ${ }^{1}$ With increasing use of digital imaging to document or screen for the presence of serious retinopathy, the reliability and reproducibility of images collected needs to be carefully examined. ${ }^{2}$ This may be especially important when a contact camera is used such as the RetCamII (Clarity Medical Systems, Pleasanton, CA, USA), which may compress the eye, thus potentially influencing retinal blood flow and affecting evaluation of the presence of plus disease in the digital image. During routine diagnostic examinations, we observed how unintentional elevation of intraocular pressure by compression with the RetCam hand-held lens and report this finding that has the potential for changing the appearance of plus disease.

\section{Case report}

A male infant born at 27 weeks of gestation and birth weight of $800 \mathrm{~g}$ was transferred to our hospital for ROP treatment at 35 weeks postmenstrual age. Stage 3 ROP with plus disease was documented using indirect ophthalmoscopy. However, fundus images taken by an experienced photographer $1 \mathrm{~h}$ later using the RetCamII failed to show vascular abnormalities in the posterior pole sufficient to be called plus disease. Although the diameter of the vessels appeared to be decreased, some arterioles nearly vanished (Figure 1a).

Imaging was repeated by a pediatric ophthalmologist within $30 \mathrm{~min}$ and by carefully positioning the lens of the RetCam within the viscous material that had been applied to the corneal surface and not touching the cornea, she obtained pictures that clearly showed plus disease with wide vessels and vessel tortuosity (Figure 1b). By applying more pressure to the camera, it was possible to repeat the artifacts.

All images were then reviewed. In addition to the initial findings, absence of the arterial blood column on the optic nerve was noticed, and the area of retinal whitening in the first picture (Figure 1a) was larger than in the second one (Figure 1b). To verify the observations, quantification with Retinal Image multiScale Analysis
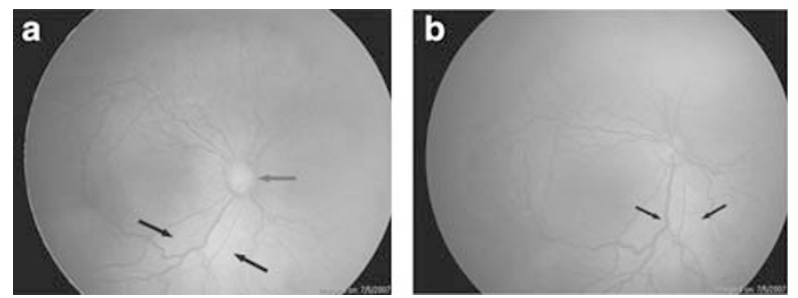

Figure 1 (a) Initial examination: Zone 2 Stage 3, with 'subtle' arterial components, note the absent blood column at the optic disc (gray arrow) and whitening of the underlying retina, which is larger than in Figure 1b (black arrows). (b) Second examination: Zone 2, threshold disease, dilatation and tortuosity of venules and arterioles in four quadrants, multiple shunts and less retinal whitening (black arrows). 